\title{
Direct Simulation of Liquid Water Dynamics in the Gas Channel of a Polymer Electrolyte Fuel Cell
}

Chaozhong Qin, Dirk Rensink, S. Majid Hassanizadeh and Stephan Fell

J. Electrochem. Soc. 2012, Volume 159, Issue 4, Pages B434-B443. doi: 10.1149/2.004205jes $\begin{array}{cl}\text { Email alerting } & \begin{array}{l}\text { Receive free email alerts when new articles cite this article - sign up } \\ \text { service }\end{array}\end{array}$

To subscribe to Journal of The Electrochemical Society go to: http://jes.ecsdl.org/subscriptions 


\title{
Direct Simulation of Liquid Water Dynamics in the Gas Channel of a Polymer Electrolyte Fuel Cell
}

\author{
Chaozhong Qin, ${ }^{\text {a,b }}$ Dirk Rensink, ${ }^{\text {a,z }}$ S. Majid Hassanizadeh,, and Stephan Fell ${ }^{\mathrm{a}}$ \\ ${ }^{a}$ Adam Opel AG, GME Engineering, GM Alternative Propulsion Europe, Rüsselsheim D-65423, Germany \\ ${ }^{b}$ Department of Earth Sciences, Utrecht University, Utrecht 3508, The Netherlands
}

\begin{abstract}
For better water management in gas channels (GCs) of polymer electrolyte fuel cells (PEFCs), a profound understanding of the liquid water dynamics is needed. In this study, we propose a novel geometrical setup to conduct a series of direct simulations of the liquid water dynamics in a GC. The conducting pathways in the gas diffusion layer (GDL) are simplified by three cylindrical pipes connected to a liquid water reservoir representing the catalyst layer (CL). The droplet dynamics, corner film dynamics, and the competition between the film and droplet flows in the GC are explored in detail. The results show that the three-phase contact line plays an important role in resisting the gas drag force for a droplet movement in the GC. The gas drag force can dominate the film flow along the GC corners, and a proper selection of the contact angle of the GC sidewalls is necessary to balance two requirements: increasing the film removal ability and removing the water clogging fast. The competing mechanisms of the droplet and film flows give us the possibility to regulate liquid water flow into GCs, and maybe lead to a better water management in GCs. Finally, the results from this work also serve to provide insights into the development of a phenomenological model for the liquid water flooding in GCs.

() 2012 The Electrochemical Society. [DOI: 10.1149/2.004205jes] All rights reserved.
\end{abstract}

Manuscript submitted November 7, 2011; revised manuscript received January 17, 2012. Published February 10, 2012.

Water plays an important role in the operation of polymer electrolyte fuel cells (PEFCs). In order to reduce ohmic resistance, the membrane needs to keep high water content. However, too much water accumulating in PEFCs would hinder reactants diffusion toward reactive sites. This is known as "water flooding". Water flooding in PEFCs gives rise to reactant starvation, increases parasitic pressure loss along the gas channel (GC), and also accelerates cell degradation. ${ }^{1-4}$

In the manufacture of PEFCs, GCs are normally grooved on both sides of bipolar plates (BPs). They have the following two main functions: (1) delivering gaseous reactants into catalyst layers (CLs), (2) providing pathways for excessive water to go out of cells. A typical $\mathrm{GC}$ is composed of hydrophilic sidewalls, and enclosed by the surface of a hydrophobic gas diffusion layer (GDL). At high current densities, the cathode side of a PEFC is prone to being flooded, since liquid water is generated from the oxygen reduction reaction (ORR) in the cathode CL, and some water is also transported from the anode to the cathode under the electro-osmotic drag (EOD). Then, the liquid water penetrates into the GCs, and consequently forms small droplets on the GDL surface, and film slugs at the corners. Fig. 1 shows various stages of emergence and disappearance of water droplets in a GC observed during operation. ${ }^{5}$ At the beginning, two small droplets preferentially emerged on the GDL surface, and later grew up. After two droplets coalesced, the liquid water wicked onto the hydrophilic GC sidewalls, and joined the film flow along the corners.

Water flooding in GCs always has a detrimental impact on the cell performance. It prevents reactants from reaching reactive sites; it increases the flooding levels in diffusion layers and the pressure losses along channels; and it facilitates transport of ionic impurities. ${ }^{6}$ Several engineering parameters can affect the liquid water removal and distribution in GCs. These include channel geometries and dimensions, wettabilities of channel sidewalls and GDL surfaces, as well as gas flow rates. In order to promote the water removal ability, a profound understanding of the liquid water dynamics in GCs is indispensible.

Because of its micro structure and mixed-wettability, the liquid water dynamics in a GC is quite complicated and sensitive to cell operating conditions. Over the past few years, both experimental and numerical studies have contributed to understanding this dynamics. Optical photography using transparent fuel cells has been widely employed to investigate the liquid water dynamic behaviors in GCs. This technique has excellent spatial and temporal resolutions depending upon the combination of optics and recording systems. ${ }^{7,8}$ However, it

${ }^{\mathrm{z}}$ E-mail: dirk.rensink@ de.opel.com often requires the modification of GCs in order to facilitate optical observations. This modification results in different material properties, which in turn affects the cell performance and water distribution. Additionally, it is rather difficult to quantify the liquid water distribution in GCs, particularly for the film flow. Ge and Wang ${ }^{9}$ optically observed the liquid water formation and transport in anode GCs, and found that condensed water could wick into the hydrophilic GDL and mitigated the anode channel flooding. Yang et al. ${ }^{5}$ found that water droplets emerged from preferential locations on the GDL surface under oversaturation of water vapor, and surface tension played a dominant role in the water removal out of the GC. When the GC sidewalls were highly hydrophilic, coalescence of water droplets and flow along the GC sidewalls were regarded as the main water removal mechanism. Zhang et al. ${ }^{10}$ also presented two models of water removal in the GC based on the magnitude of gas inlet velocity. When the gas velocity was high, the water droplet size was small and mainly swept by the drag force. At the low gas flow rate, emerged droplets would grow to a critical size comparable to the channel dimensions, and then wicked into the sidewalls under the capillary forces. In addition, the authors fitted a semi-empirical relationship between the droplet detachment diameter and the gas velocity. Most recently, Hussaini and Wang ${ }^{11}$ presented a flow map illustrating various two-phase flow patterns in GCs. They introduced a parameter called wetted area ratio to quantify the liquid water coverage effect on the GDL surface, which would affect the liquid water distribution and transport inside the GDL, as well as hinder reactants diffusion into reactive sites.

Numerically, several interface-tracking methods can be conveniently employed to investigate the liquid water behaviors in micro GCs. Theodorakakos et al. ${ }^{12}$ used the volume of fluid (VOF) method to study the detachments of water droplets from different porous material surfaces used in PEFCs. Cai et al. ${ }^{13}$ utilized the VOF method in FLUENT package to study the water droplet and film behaviors in the GCs of a PEFC. It was reported that a hydrophilic channel side wall with a hydrophobic MEA surface could avoid water accumulation on the GDL surface. Rensink et al. ${ }^{14}$ utilized VOF method to investigate the behavior of a water droplet in the GC and its interaction with channel walls under pure capillary action. Zhu et al. ${ }^{15}$ also adopted the VOF method in FLUENT to study the dynamic process of a water droplet emerging from a GDL pore. Various parametric simulations including the effects of gas flow velocity, water injection velocity and dimensions of the pore were performed with a highlight on the effect of the wettabilities of GDL surface and micro channel sidewalls. Besides the widely applied VOF method, Lattice Boltzmann Method (LBM) is another effective numerical approach to study the 


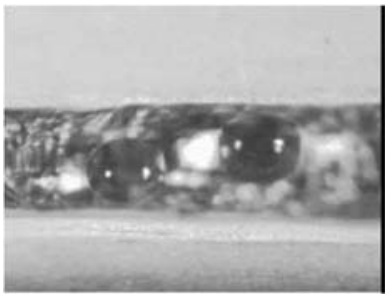

0 s

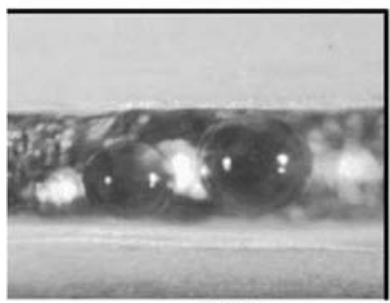

1208

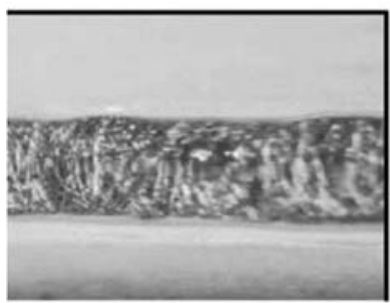

4858

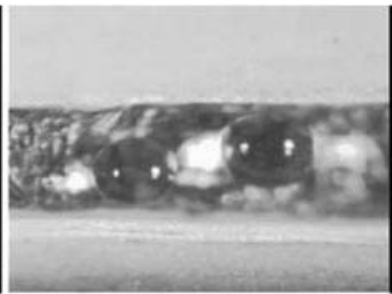

$30 \mathrm{~s}$

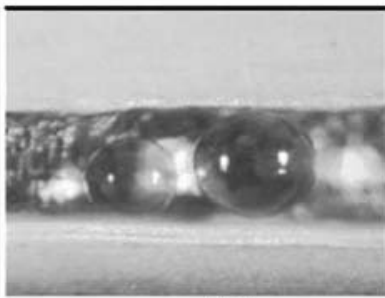

$180 \mathrm{~s}$

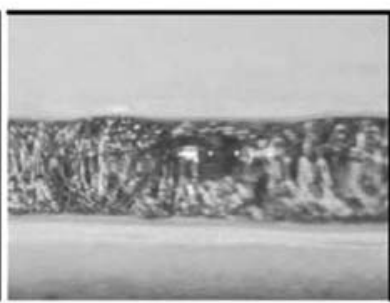

5108

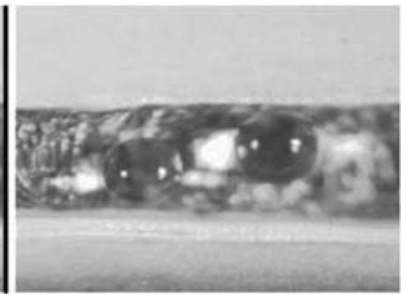

$60 \mathrm{~s}$

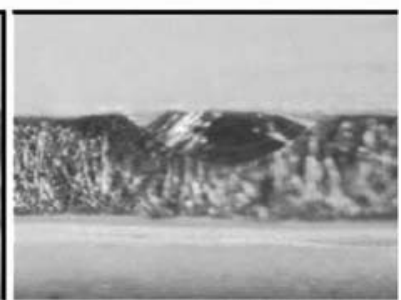

$480 \mathrm{~s}$
Figure 1. Dynamics of water droplets in a gas channel observed in experiments at the current density of $0.82 \mathrm{~A} \mathrm{~cm}^{-2}$ and the temperature of $70^{\circ} \mathrm{C}$ [Reference 5, reproduced by permission of ECS-The Electrochemical Society]. two-phase flow phenomena in micro GCs. Hao and Cheng ${ }^{16}$ used the multiphase free-energy LBM to study the formation and subsequent movement of a water droplet on the GDL surface. Recently Choi and Son ${ }^{17}$ employed the level set method to investigate the droplet motion in a micro channel with different contact angles.

Aside from extensive experimental and numerical studies, a few researchers ${ }^{6,18}$ proposed some simplified analytical models to predict the onset of liquid water droplet instability in a GC. However, they all ignored the effect of the GC width in the approximation of the velocity profile over the droplet. So, the results from these analytical models would overestimate the water droplet instabilities.

Most of previous numerical studies only focused on the droplet dynamics in GCs. However, at low gas flow rates, the film flow could be the main transport mechanism for the liquid water along GC corners. If water droplets emerge in the vicinity of the hydrophilic sidewalls of a GC, the film flow at the corners would form immediately under the capillary force. On the other hand, for the droplets emerged near the center of the GC, most of them would grow up and coalesce with each other to reach a critical size, and finally wick onto the hydrophilic sidewalls. So it is very important to investigate the film dynamics in GCs, which is explored in this work in detail.

Since the GDL has complex fibrous structure, it is difficult and time-consuming to simulate the water movement inside the GDL at the pore scale. LBM can resolve liquid water dynamics in the realistic GDL. However, to reduce computational efforts and stabilize calculations, arbitrary values have been used for both water density and viscosity in previous numerical studies, ${ }^{31,32}$ which may impact interpretations of obtained numerical results. Hence, most researchers ${ }^{15-17}$ simply "planted" certain number of droplets inside GCs or imposed a water inlet boundary condition at a small region of GDL surface. They did not include the GDL in their simulations. So, the receding phenomenon of the liquid water at the GC-GDL interface could not be modeled numerically. ${ }^{19,20}$

In this work, we account for the presence of GDL in a simple fashion. We assume that liquid water is transported from a water reservoir to the GC by three cylindrical pipes. These pipes are regarded as the formed conducting pathways for the liquid water transport through the GDL, and the water reservoir could be assumed to be the CL providing constant water flow rates. Based on this novel geometrical setup, we also investigate the competition between the droplet and film flows inside the GC, which often occurs in an operating PEFC.

The objective of this work is three-fold: (1) to explore the water droplet and film dynamics in a GC, addressing the effects of gas flow rate and wettabilities of channel sidewalls and GDL surface on the liquid water distribution and water removal; (2) to illustrate the competing mechanisms of the liquid water flow from three distinct water conducting pathways in the GDL to the GC; (3) to provide insights into the development of a phenomenological model for the water flooding in GCs.

\section{Numerical Method}

Computational domain. - In order to make the direct simulation of water dynamics in a GC feasible, we need to simplify the CL and GDL considerably. Up to now, the exact liquid water transport mechanism in the GDL is still unclear mainly due to its complex pore-structure and mixed-wettability. Nam and Kaviany ${ }^{21}$ first proposed the branchingtype flow structure, which is composed of larger main streams and smaller ones, connected to homogenously distributed condensation sites. Later, Litster et al. ${ }^{22}$ used a novel fluorescence microscopy technique to visualize the liquid water transport in the GDL, and concluded that the water transport was dominated by fingering and channeling, not a converging capillary tree. Recently, Hartnig et al. ${ }^{20}$ also observed a similar transport process in the GDL based on the high-resolution synchrotron X-ray radiography. So, in this work, it is quite reasonable to simplify the formed conducting pathways in the GDL by means of three capillary channels (i.e. pipes) as shown in Fig. 2. Two cylindrical pipes are located in the vicinity of two sidewalls of the GC for simulating the film flow along the corners, and the third cylindrical 
(a)

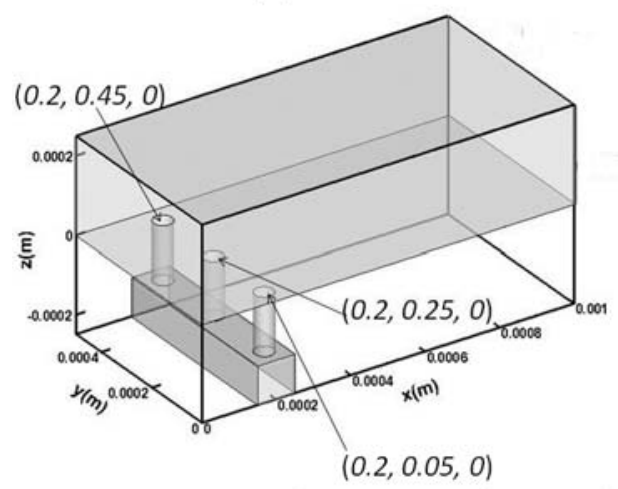

(b)

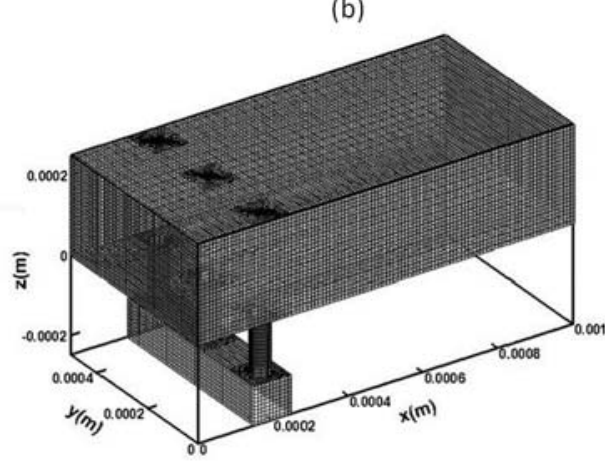

Circle center coordinates with unit, $\mathrm{mm}$

Figure 2. (a) Computational domain and (b) the corresponding mesh.

pipe is mounted at the center for the droplet dynamic study. The exact positions of these three pipes are marked in Fig. 2a.

The CL is simplified by a water reservoir for providing the liquid water. We do not consider the coalescence of droplets in the GC, and the dynamics of only one droplet is studied in this work. Therefore, we use a relatively short channel with the length of $1 \mathrm{~mm}$ to reduce computational efforts. At last, we note that liquid water dynamics in the GC also depends on the surface texture of the GDL. This is not included in the present work for simplicity, which could be our further studies.

Governing equations. - The VOF method ${ }^{23,24}$ is used in this work to explicitly track the interface between the gas and liquid water. We assume to have unsteady-state, isothermal, laminar three-dimensional flow in the GC and no phase change. The densities of both phases are assumed to remain constant due to the small pressure gradients.

In the VOF method, each computational element may have only one or both fluid phases. So, we introduce a phase indicator $a$ with the definition:

$$
a=\frac{V_{l}}{V_{c v}}
$$

where $V_{l}$ is the volume of the liquid phase in a computational element, and $V_{c v}$ is the volume of the computational element.

Based on the averaging theorem, ${ }^{25,26}$ we can write down the governing equations as follows:

$$
\nabla \cdot \vec{v}=0
$$

$$
\frac{\partial(\rho \vec{v})}{\partial t}+\nabla \cdot(\rho \vec{v} \vec{v})=-\nabla p+\mu \nabla^{2} \vec{v}+\frac{1}{\delta V_{c v}} \int_{\delta S}\left(p_{l}-p_{g}\right) \vec{n}_{l} d s_{\xi}
$$

$$
\frac{\partial a}{\partial t}+\vec{v} \cdot \nabla a=0
$$

Here, $\rho, \mu$, and $p$ are called mixture density, mixture viscosity and mixture pressure, respectively. They have the following definitions:

$$
\begin{gathered}
\rho=a \rho_{l}+(1-a) \rho_{g} \\
\mu=a \mu_{l}+(1-a) \mu_{g} \\
p=a p_{l}+(1-a) p_{g}
\end{gathered}
$$

where subscripts $l$ and $g$ stand for liquid and gas, respectively.

Eq. 3 is the momentum equation of the mixture gas-water flow. The gravity is neglected due to the quite small Bond number

$\left(B o=\left(\rho_{l}-\rho_{g}\right) g L^{2} / \sigma\right)$. The last term in Eq. 3 represents the effect of the capillary force at the interface between gas and liquid water, $\delta S$ denotes the interface area, $\vec{n}_{l}$ is the unit normal vector pointing outwards the liquid phase. Brackbill et al. ${ }^{27}$ first assumed that the interface between phases could be represented by a transition region with finite thickness, and the indicator $a$ varied continuously across this region. Then they used the continuum method to convert the capillary force to a volumetric force, as follows:

$$
F=\frac{1}{\delta V_{c v}} \int_{\delta S}\left(p_{l}-p_{g}\right) n_{l} d s_{\xi}=\frac{2 \sigma \rho k \nabla a}{\rho_{l}+\rho_{g}}
$$

where $\sigma$ is the surface tension, $\rho$ is the mixture density, and $k$ denotes the converted curvature, which is defined as:

$$
k=\nabla \cdot \vec{n}=\nabla \cdot\left(\frac{\nabla a}{|\nabla a|}\right)
$$

where $\vec{n}$ is the unit vector normal to the indicator field.

The wall adhesion is also considered in this work; the unit normal vector at the cell next to the wall is given as: ${ }^{28}$

$$
\vec{n}=\vec{n}_{w} \cos \theta+\vec{t}_{w} \sin \theta
$$

where $\vec{n}_{w}$ and $\vec{t}_{w}$ denote the unit vectors normal and tangential to the solid wall, respectively, and $\theta$ is the contact angle of the solid wall.

Note that, in the derivation of governing equations, both phases are assumed to share the same velocity in each computational element. Therefore, the VOF method neglects the slip phenomenon between the gas and liquid water. We restrict our studies to the water dynamics in GCs in automotive applications. ${ }^{2}$ The corresponding physical parameters for typical operating conditions are listed in Table I. The densities and viscosities of both phases are given at a typical operating temperature of $353.15 \mathrm{~K}$.

\section{Table I. Geometrical and physical parameters.}

Parameters

Values

GC width/height/length

Height of pipes

Bath width/height/length

Gas density, $\rho_{g}$

Liquid water density, $\rho_{l}$

Gas dynamic viscosity, $\mu_{g}$

Liquid water dynamic viscosity, $\mu_{l}$

Surface tension, $\sigma$
$0.5 / 0.25 / 1.0 \mathrm{~mm}$

$0.15 \mathrm{~mm}$

$0.5 / 0.1 / 0.1 \mathrm{~mm}$

$2.0016 \mathrm{~kg} \mathrm{~m}^{-3}$

$972 \mathrm{~kg} \mathrm{~m}^{-3}$

$2.03 \times 10^{-5} \mathrm{~kg} \mathrm{~m}^{-1} \mathrm{~s}^{-1}$

$3.5 \times 10^{-4} \mathrm{~kg} \mathrm{~m}^{-1} \mathrm{~s}^{-1}$

$0.0625 \mathrm{~N} \mathrm{~m}^{-1}$ 
Table II. Simulated cases in this work.

\begin{tabular}{|c|c|c|c|c|c|c|}
\hline Case name & $\begin{array}{l}\text { Gas } \\
\text { inlet Re } \\
\text { number }\end{array}$ & $\begin{array}{l}\text { Mass flow } \\
\text { rate of } \\
\text { liquid water } \\
\left(\mathrm{kg} \mathrm{s}^{-1}\right)\end{array}$ & $\begin{array}{c}\text { Contact } \\
\text { angle of GC } \\
\text { sidewalls } \\
\text { (degrees) }\end{array}$ & $\begin{array}{c}\text { Contact } \\
\text { angle of GC } \\
\text { down wall } \\
\text { (degrees) }\end{array}$ & $\begin{array}{l}\text { Diameter of } \\
\text { side pipes } \\
\quad(\mu \mathrm{m})\end{array}$ & $\begin{array}{l}\text { Diameter of } \\
\text { middle pipe } \\
\qquad(\mu \mathrm{m})\end{array}$ \\
\hline Case1_droplet & 150 & $2.0 \times 10^{-6}$ & 40 & 140 & None & 50 \\
\hline Case2_droplet & 200 & $2.0 \times 10^{-6}$ & 40 & 140 & None & 50 \\
\hline Case3_droplet & 250 & $2.0 \times 10^{-6}$ & 40 & 140 & None & 50 \\
\hline Case4_droplet & 300 & $2.0 \times 10^{-6}$ & 40 & 140 & None & 50 \\
\hline Case7_droplet & 150 & $2.0 \times 10^{-6}$ & 40 & 170 & None & 50 \\
\hline Case8_film & 150 & $2.0 \times 10^{-6}$ & 40 & 140 & 50 & None \\
\hline Case9_film & 200 & $2.0 \times 10^{-6}$ & 40 & 140 & 50 & None \\
\hline Case10_film & 250 & $2.0 \times 10^{-6}$ & 40 & 140 & 50 & None \\
\hline Case11_film & 150 & $2.0 \times 10^{-6}$ & 0 & 140 & 50 & None \\
\hline Case12_film & 150 & $2.0 \times 10^{-6}$ & 20 & 140 & 50 & None \\
\hline Case17_both & 150 & $2.0 \times 10^{-6}$ & 40 & 140 & 50 & 40 \\
\hline
\end{tabular}

Boundary and initial conditions. - Uniform gas inlet velocities normal to the $\mathrm{GC}$ inlet $(\mathrm{x}=0.0 \mathrm{~mm})$ are specified for different cases shown in Table II. They are related to the corresponding Reynolds numbers as follows:

$$
v_{i n}=\operatorname{Re} \frac{\mu_{g}}{\rho_{g} D_{g c}}
$$

Here, Re is the Reynolds number, and $D_{g c}$ is the hydraulic diameter of the GC, defined as:

$$
D_{g c}=\frac{4 A_{g c}}{L_{g c}}
$$

where $A_{g c}$ is the cross-sectional area of the GC and $L_{g c}$ is the wetted perimeter of the cross-section.

A pressure outlet boundary condition is imposed at the GC outlet (x $=1 \mathrm{~mm})$. At the inlet boundary of the CL reservoir $(\mathrm{z}=-0.25 \mathrm{~mm})$, a constant mass flow rate of liquid water is specified. The contact angle in the reservoir is assumed to be zero. For the rest of the boundaries, the no-slip wall condition with a specified static contact angle is employed.

Before the calculation of each case, we use the inlet velocity to initialize the flow field, and the pipes are fully filled with liquid water to reduce computational efforts.

Mesh and numerical implementation.- Adequate mesh density is essential to capture sharp interfaces and reduce smeared regions in VOF simulations. ${ }^{23}$ The basic mesh used in this work is shown in Fig. 2b. They are mainly cubic cells with dimensions of $10 \times 10$ $\times 10 \mu \mathrm{m}$ except for the refined regions nearby the pipes. The mesh independency study is also done by increasing and decreasing the cell size by $20 \%$ and $40 \%$, respectively. ${ }^{15}$ The obtained results for water dynamics and flow field are found to be quite similar.

The set of transient governing equations and boundary conditions given above are discretized by finite volume method with secondorder schemes based on a commercial CFD solver FLUENT 12.0.16. Eq. 4 describes the evolution of the volume fraction of liquid water, which is a hyperbolic partial differential equation (PDE). So, its discretization is crucial to VOF simulations. In this work, we use the geometric reconstruction scheme, which represents the interface between both phases by a piecewise-linear curve. It works quite well on the orthogonal cells.

For transient VOF simulations, the selected time step can affect the numerical results and computational efforts much. A big time step would give rise to smeared interfaces and even unreliable results, while a small time step increases computational efforts. In this work, in order to balance the two effects, we choose time steps such that the global Courant number ${ }^{28}$ is less than 0.6. For all the simulated cases, the scaled residuals of all the equations are less than $10^{-5}$.

\section{Results and Discussion}

Seventeen different cases are conducted in this work to explore the liquid water dynamics in a GC. The corresponding boundary conditions and pipe diameters are given in Table II. Cases (1-7) are used to study the water droplet dynamics including the effects of the gas flow rate represented by Reynolds ( $\mathrm{Re})$ number, and the contact angle of GDL surface. Cases (8-14) are used to study the film dynamics in the GC taking into account the effects of the Re number and the contact angle of GC sidewalls. The remaining cases are meant to illustrate the competing mechanisms of the droplet and film flows. Note that in PEFCs a practical gas inlet velocity of $5 \mathrm{~m} / \mathrm{s}$ can be used, which corresponds to a Re number of about 164 in this study. Based on the simulations in this work, we attempt to answer the following questions:

(1) What is the role of the three-phase contact line in a droplet detachment?

(2) Can increasing the hydrophobicity of the GDL surface enhance the water removal ability?

(3) How do the gas drag and surface tension forces affect the film flow along the GC corners?

(4) Is decreasing the hydrophilicity of the GC sidewalls beneficial to the water removal?

(5) How do the conducting pathways formed in the GDL compete to transport liquid water into the GC?

We should point out that the liquid water flow rate used in this work is too large compared to real situations. But, up to now, this has been unavoidable in all interface-tracking VOF simulations in the context of PEFC applications. In order to reduce computational efforts, the mass flow rate has to be enlarged dramatically to make numerical study feasible. However, in the film flow of case 14, we reduce the mass flow of the liquid water four times to address its effect on the film dynamics in the GC. 
(a) $\mathrm{t}=0.5 \mathrm{~ms}$ Volume fraction of liquid water

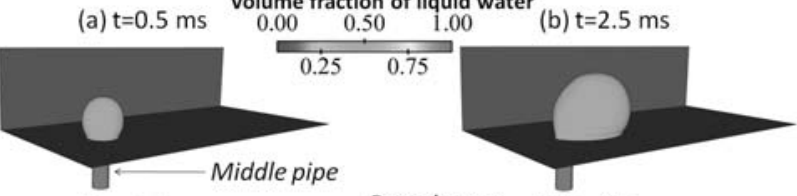

(c) $\mathrm{t}=3.5 \mathrm{~ms}$

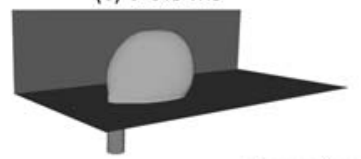

Detachment $\quad$ (d) $\mathrm{t}=4.6 \mathrm{~ms}$

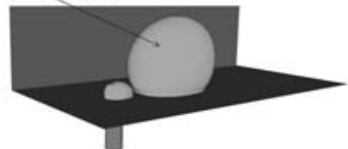

(e) $\mathrm{t}=5.0 \mathrm{~ms}$ Spreading over the sidewalls ${ }_{(\mathrm{f}) \mathrm{t}=6.0 \mathrm{~ms}}$

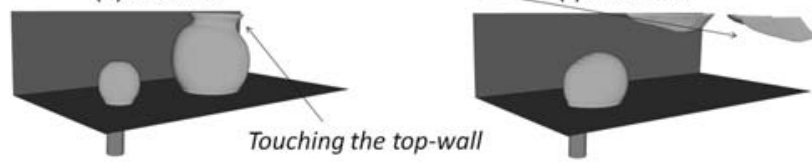

(g) Three-phase contact line movement with time

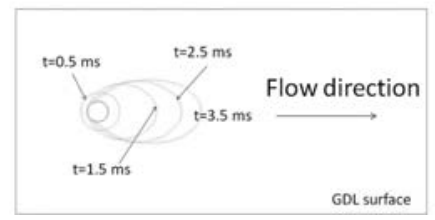

Figure 3. Dynamic progress of one water droplet emergence, growth, detachment, and touching the top wall (case 1: gas Re number, 150; water flow rate, $2.0 \times 10^{-6} \mathrm{~kg} \mathrm{~s}^{-1}$; sidewall contact angle, $40^{\circ}$; GDL surface contact angle, $140^{\circ}$; middle pipe diameter, $50 \mu \mathrm{m}$ ).

Droplet dynamics. - Fig. 3 shows the dynamic process of one water droplet movement along the GC (case 1). Since only one droplet is focused here, the two side pipes are not included in the simulation. At $\mathrm{t}=0.5 \mathrm{~ms}$ (Fig. 3a), a small droplet forms on the hydrophobic GDL surface. Due to the relatively small gas drag force, the deformation of the droplet is initially very small. With the continuous water filling through the pipe, the droplet becomes bigger and more deformable (Fig. $3 \mathrm{~b}$ and $3 \mathrm{c}$ ). The length of three-phase contact line along the GC increases with time until the detachment of the droplet occurs (Fig. 3g). This leads to an increase of adhesion force, which resists the increased gas drag force. Another parameter, called droplet contact angle hysteresis, also increases the adhesion force along the contact line. The contact angle hysteresis may depend on gas flow rate, droplet size, roughness of the GDL surface, and channel dimensions in a complex way. ${ }^{6}$ We have chosen to neglect this effect, and only a fixed static contact angle is assumed in this work. As a result, the increased adhesion force comes only from the contact line deformation as shown in Fig. 3g. At about $\mathrm{t}=4.6 \mathrm{~ms}$ (Fig. 3d), we observe the detachment of the droplet. Note that we claim that the droplet is detached by the gas drag force in this work, when the droplet contact line totally separates from the pipe inlet circumference at the GDL surface. At $\mathrm{t}=5.0 \mathrm{~ms}$ (Fig. 3e) the detached droplet suddenly touches the GC top wall, and we also find that the deformation of the droplet is much smaller than in Fig. 3c. After touching the hydrophilic top wall of the $\mathrm{GC}$, the liquid water wicks into the corners and the top wall under the surface tension force. So, when water clogging happens in GCs of an operating PEFC, highly hydrophilic sidewalls are preferred to spread out liquid water quickly.

When the gas flow rate is increased (case 3), the droplet dynamic process is shown in Fig. 4. The detachment of the droplet occurs much earlier than in Fig. 3. In Fig. 4d, a small water tail behind the droplet is observed, which may be related to the increased gas flow rate. From the comparison of Fig. 3 and Fig. 4, we conclude that the droplet deformation increases with an increase of the gas flow rate. The critical times at which the detachments of droplets occur are plotted in Fig. 5 for four different Re numbers. Since a constant water flow rate is assumed, the critical time directly represents the critical droplet size in the GC. As seen from Fig. 5, increasing the gas flow (a) $\mathrm{t}=0.5 \mathrm{~ms}$

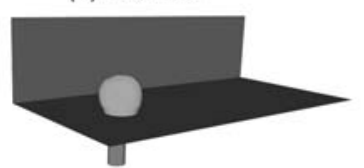

(c) $\mathrm{t}=1.3 \mathrm{~ms}$

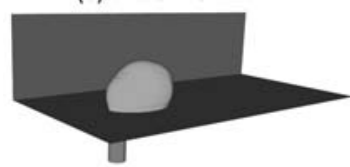

(e) $\mathrm{t}=2.2 \mathrm{~ms}$

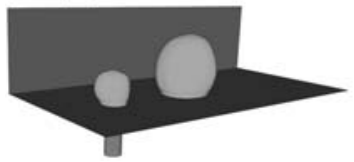

(b) t=1.0 ms

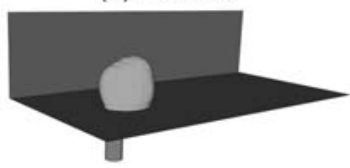

(d) $\mathrm{t}=1.6 \mathrm{~ms}$

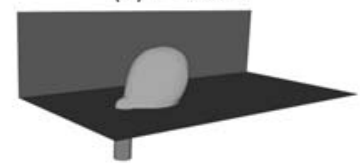

(f) $\mathrm{t}=2.6 \mathrm{~ms}$

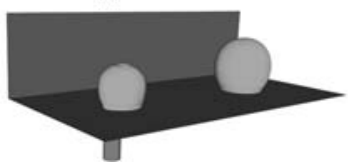

Figure 4. Dynamic progress of one water droplet emergence, growth and detachment (case 3: gas Re number, 250; water flow rate, $2.0 \times 10^{-6} \mathrm{~kg} \mathrm{~s}^{-1}$; sidewall contact angle, $40^{\circ}$; GDL surface contact angle, $140^{\circ}$; middle pipe diameter, $50 \mu \mathrm{m})$.

rate can assist in the droplet removal in the GC. However, this effect becomes much weaker after the Re number is larger than 250 , which means that the force balance for a droplet ${ }^{18}$ is highly nonlinear.

Fig. 6 shows the droplet dynamics and its contact line evolution with time in the cross-section $(\mathrm{y}=0.25 \mathrm{~mm})$ of the $\mathrm{GC}$ for case 2 . Before the droplet detachment (Fig. 6a-6c), the gas drag force exerted on the droplet becomes bigger and bigger due to the increase of the droplet size. This force is resisted by the adhesion along the contact line, which extends in both longitudinal and transversal directions (Fig. 6f). In addition, the droplet deforms more and more. After the detachment (Fig. 6e), the gas drag force imposed on the droplet decreases dramatically due to the fast movement of the droplet along the GC. As a result, the contact line shape approaches a circle (Fig. 6f), indicative of the reduced adhesion force.

Fig. 7 shows the droplet dynamics on a hydrophilic GDL surface (case 5). As expected, large water coverage is found during the whole process. No distinct droplet detachment can be identified. In fact it behaves more like a film flow spreading over the surface. Therefore, hydrophobic GDLs are commonly used in PEFCs to circumvent heavy water accumulation and large water coverage. Fig. 8 illustrates the droplet dynamics on a slightly hydrophobic GDL (case 6). It is interesting to find that the critical time for the droplet detachment $(\mathrm{t}=2.5 \mathrm{~ms})$ is much shorter compared to that in Fig. $3(\mathrm{t}=4.4 \mathrm{~ms})$.

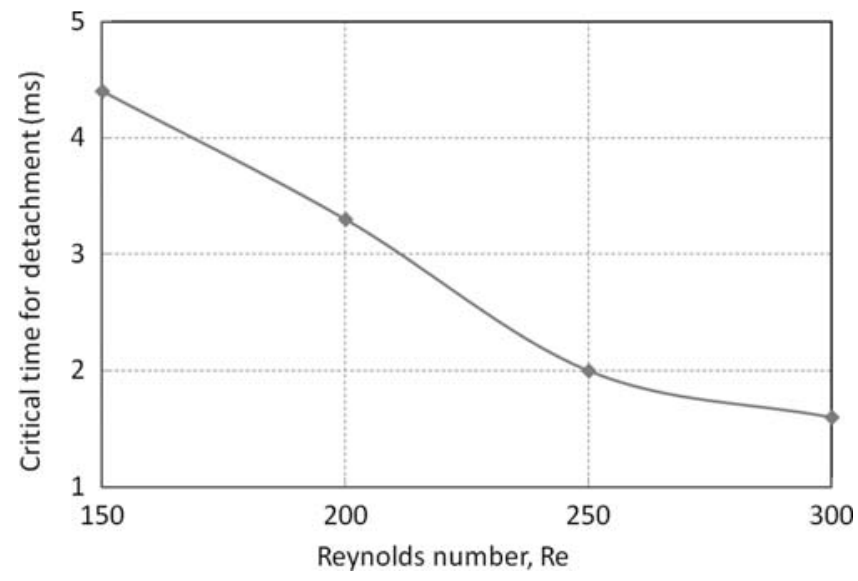

Figure 5. Critical time for the detachment of a water droplet vs. Reynolds number. 
(a) $\mathrm{t}=1.0 \mathrm{~ms}$

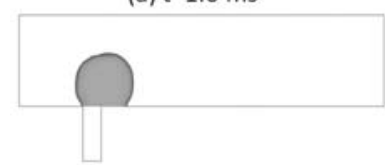

(c) $\mathrm{t}=2.8 \mathrm{~ms}$

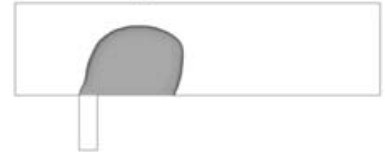

(e) $\mathrm{t}=3.6 \mathrm{~ms}$

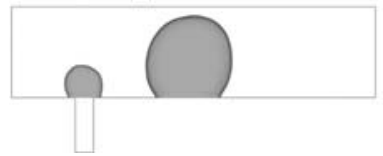

(b) $\mathrm{t}=2.0 \mathrm{~ms}$

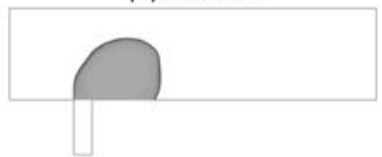

(d) $\mathrm{t}=3.3 \mathrm{~ms}$

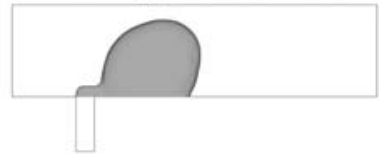

(f) Contact line movement

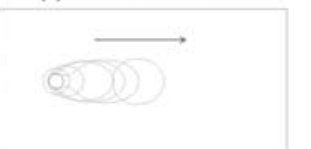

Figure 6. Droplet dynamics and its contact line movement with time in the cross-section $(\mathrm{y}=0.25 \mathrm{~mm}$ ) of the GC (case 2: gas Re number, 200; water flow rate, $2.0 \times 10^{-6} \mathrm{~kg} \mathrm{~s}^{-1}$; sidewall contact angle, $40^{\circ}$; GDL surface contact angle, $140^{\circ}$; middle pipe diameter, $50 \mu \mathrm{m}$ ).

This can be explained by the fact that the contact line deformation on this slightly hydrophobic GDL is very small as it cannot resist the increased gas drag force (Fig. 9a). As a result, the droplet is detached at a quite early stage. Fig. 9 shows the contact line comparison between case 5 (contact angle of GDL surface, 110 degrees) and case 7 (170 degrees). It is evident that the contact area in case 5 is bigger than in case 7 at the same moment due to its slightly hydrophobic GDL. In addition, we find that the contact line deformation in case 7 is much larger than in case 5 , which has the ability to resist the increased gas drag force. We conclude that the smaller the contact angle of the hydrophobic GDL surface, the smaller the contact line deformation under the same flow condition will be. However, as mentioned above, in this study we do not consider the effect of contact angle hysteresis due to the VOF model limitation. Recently, Kumbur et al. $^{6}$ has experimentally demonstrated that the contact angle hysteresis of one droplet in the GC increased as increasing the GDL hydrophobicity. Since the contact angle hysteresis also contributes to increasing the droplet adhesion force, we could state that a slightly hydrophobic GDL is favorable to droplet detachment. It needs to be pointed out that the hydrophobic GDL with a smaller contact angle results in large water coverage over the surface, which is disadvantageous to (a) $\mathrm{t}=0.5 \mathrm{~ms}$

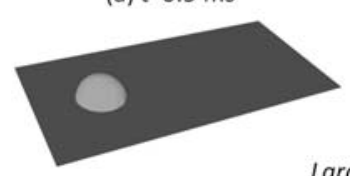

(c) $\mathrm{t}=2.7 \mathrm{~ms}$

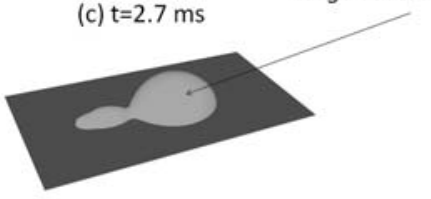

(e) $\mathrm{t}=4.0 \mathrm{~ms}$

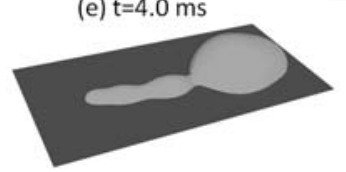

(b) $\mathrm{t}=1.5 \mathrm{~ms}$

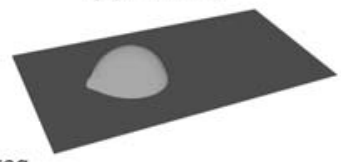

(d) $\mathrm{t}=3.5 \mathrm{~ms}$
Figure 7. Droplet dynamics on a slightly hydrophilic GDL surface (case 5: gas Re number, 150; water flow rate, $2.0 \times 10^{-6} \mathrm{~kg} \mathrm{~s}^{-1}$; sidewall contact angle, $40^{\circ}$; GDL surface contact angle, $80^{\circ}$; middle pipe diameter, $50 \mu \mathrm{m}$ ).

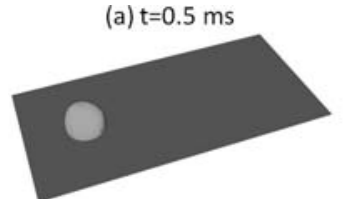

(b) $\mathrm{t}=1.0 \mathrm{~ms}$

$\begin{array}{lll}\text { (c) } \mathrm{t}=1.5 \mathrm{~ms} \text { Relatively small deformation } & \text { (d) } \mathrm{t}=2.0 \mathrm{~ms}\end{array}$

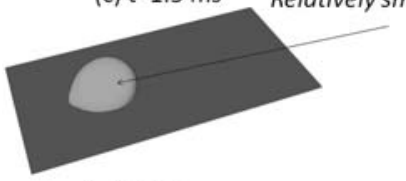

(e) $\mathrm{t}=2.5 \mathrm{~ms}$
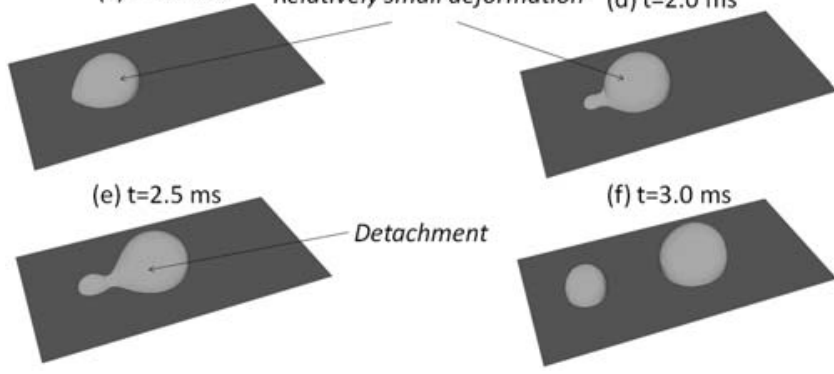

(f) $\mathrm{t}=3.0 \mathrm{~ms}$

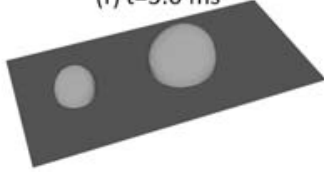

Figure 8. Dynamic progress of one water droplet emergence, growth and detachment (case 6: gas Re number, 150; water flow rate, $2.0 \times 10^{-6} \mathrm{~kg} \mathrm{~s}^{-1}$; sidewall contact angle, $40^{\circ}$; GDL surface contact angle, $110^{\circ}$; middle pipe diameter, $50 \mu \mathrm{m}$ ).

reactants diffusion into reactive sites. When the contact angle of the GDL surface is increased to 170 degrees (case 7), the critical time is found to be about $4.5 \mathrm{~ms}$, which is quite close to the critical time for the detachment in Fig. 3 (4.4 ms). This means that highly hydrophobic GDL surface is unnecessary for the droplet removal in the GC. However, it can reduce the water coverage over the GDL surface.

Film dynamics. - At low gas flow rates, film flow could be the main transport mechanism for the liquid water removal in GCs. As shown in Fig. 3f, when the droplet reaches its critical size comparable to the GC dimensions, it joins the film flow along the corners. There is another mechanism that contributes to the film filling, namely, the capillary force of the hydrophilic sidewalls of the GC. This contribution is

(a)

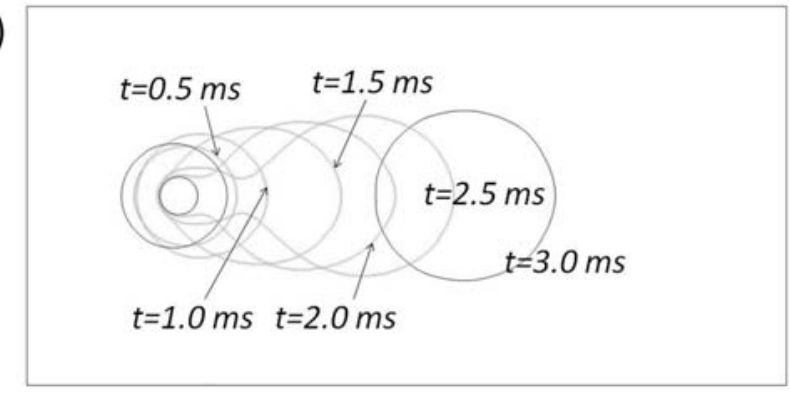

(b)

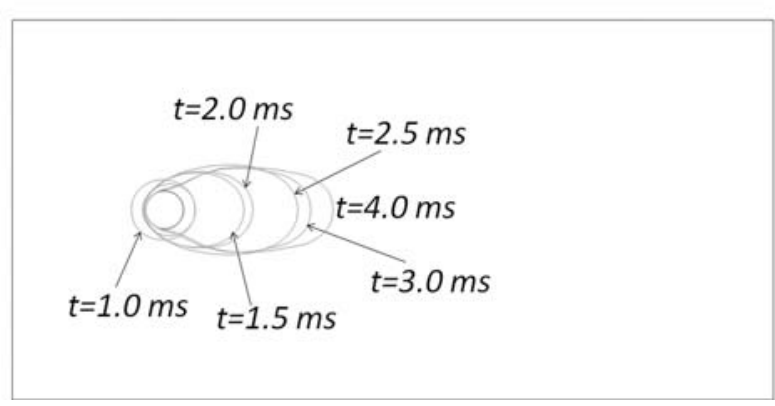

Figure 9. (a) Contact line movement with time (case 6: gas Re number, 150; water flow rate, $2.0 \times 10^{-6} \mathrm{~kg} \mathrm{~s}^{-1}$; sidewall contact angle, $40^{\circ}$; GDL surface contact angle, $110^{\circ}$; middle pipe diameter, $\left.50 \mu \mathrm{m}\right)$; (b) contact line movement with time (case 7: gas Re number, 150; water flow rate, $2.0 \times 10^{-6} \mathrm{~kg} \mathrm{~s}^{-1}$; sidewall contact angle, $40^{\circ}$; GDL surface contact angle, $170^{\circ}$; middle pipe diameter, $50 \mu \mathrm{m})$. 
(a) $\mathrm{t}=0.1 \mathrm{~ms}$

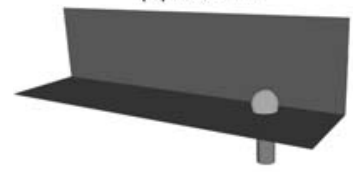

(c) $\mathrm{t}=1.0 \mathrm{~ms}$

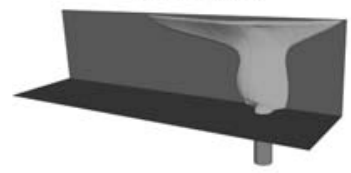

(e) $\mathrm{t}=3.0 \mathrm{~ms}$

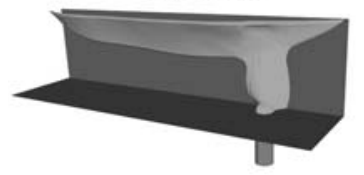

(g) $\mathrm{t}=6.0 \mathrm{~ms}$

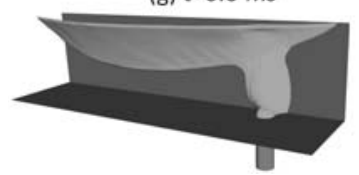

(b) $\mathrm{t}=0.5 \mathrm{~ms}$

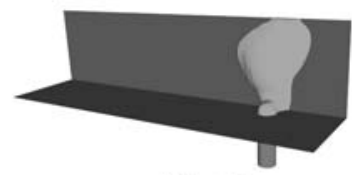

(d) $\mathrm{t}=2.0 \mathrm{~ms}$

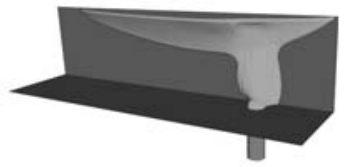

(f) $\mathrm{t}=4.5 \mathrm{~ms}$

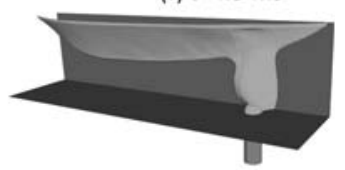

Figure 10. Dynamics of film flow along the corner of the GC (case 8: gas Re number, 150; water flow rate, $2.0 \times 10^{-6} \mathrm{~kg} \mathrm{~s}^{-1}$; sidewall contact angle, $40^{\circ}$; GDL surface contact angle, $140^{\circ}$; side pipe diameter, $\left.50 \mu \mathrm{m}\right)$.

discussed in this section. In order to reduce the computational efforts, we take advantage of the symmetrical film flow in the GC, and use only half of the GC as our computational domain. In these computations, the middle pipe pertaining to the droplet formation is neglected. At $t$ $=0.1 \mathrm{~ms}$ (Fig. 10a), a small water droplet forms on the hydrophobic GDL surface nearby the sidewall. When the droplet grows, at some critical size, it touches the GC sidewall, and spreads over the sidewall quickly with the help of the surface tension force (Fig. 10b). With further feeding of the liquid water into the $\mathrm{GC}$, the film flow forms along the corner of the GC as shown in Fig. 10d. From the water inlet position (side pipe), the water spreads toward the GC outlet and the GC inlet, with the film thickness reducing in both directions. This is attributed to the fact that the surface tension dominates the film flow at the early stage. However, the gas drag force plays a more and more important role as more and more liquid water accumulates in the GC. Finally, we get a totally different film shape from the water inlet position to the GC outlet, which is from the thin to the thick. This indicates that the gas drag force begins to dominate the film flow compared with the surface tension force (Fig. 10g). Note that, at the outlet region, the film shape changes a little, perhaps due to the boundary effect.

As mentioned earlier, in our simulations, we specify a much larger liquid water flow rate $\left(2 \mathrm{e}-06 \mathrm{~kg} \mathrm{~s}^{-1}\right)$ from the CL than in real situations. This unrealistic water flow rate may render our numerical results unrealistic. In order to address this issue, we reduce the water flow rate by a factor four and the obtained film dynamic process is shown in Fig. 11. As expected, in comparison to the results shown in Fig. 10, the water filling time is much longer for reaching a certain value of water saturation in the whole GC. The second difference can be seen from Fig. 11b. After the grown-up droplet touches the sidewall of the GC, the discontinuity of the water flow is observed due to the slow water filling rate from the side pipe (i.e. small water flow rate). The isolated water film at the corner flows toward the GC outlet under the gas drag and surface tension forces (Fig. 11e). The film shape distribution also shows the same tendency as in Fig. 10g, which implies that the gas drag force plays a dominant role in the film flow. When the second water droplet reaches its critical size, it also touches the sidewall and fills the film flow again as shown in Fig. 11f. This water refilling phenomenon demonstrates that the film flow at the corner has a periodic feature

(a) $\mathrm{t}=0.86 \mathrm{~ms}$

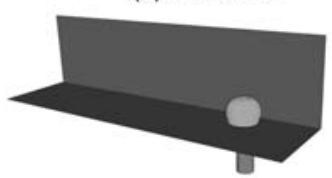

(c) $\mathrm{t}=3.74 \mathrm{~ms}$

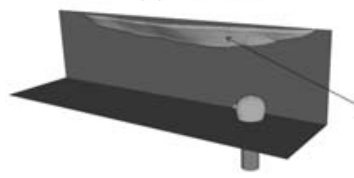

(e) $t=7.03 \mathrm{~ms}$

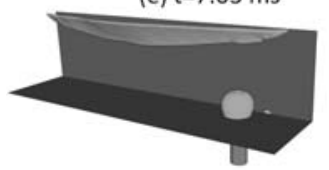

(g) $\mathrm{t}=13.2 \mathrm{~ms}$

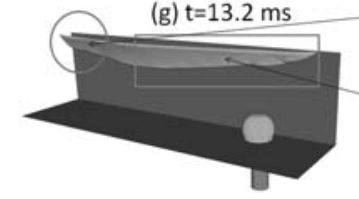

Boundary effect

Film thickness is from thin to thick due to the gas drag force. (b) $\mathrm{t}=1.27 \mathrm{~ms}$

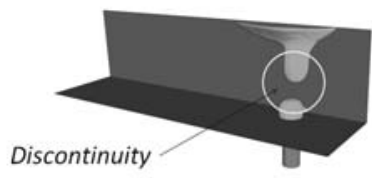

(d) $\mathrm{t}=5.8 \mathrm{~ms}$

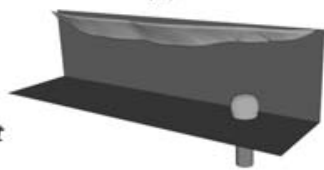

(f) $\mathrm{t}=10.32 \mathrm{~ms}$

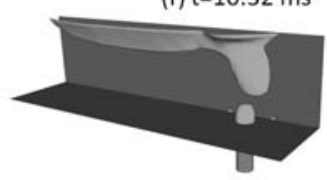

${ }_{0}$
Figure 11. Dynamics of film flow along the corner of the GC (case 14: gas Re number, 150 ; water flow rate, $5.0 \times 10^{-7} \mathrm{~kg} \mathrm{~s}^{-1}$; sidewall contact angle, $40^{\circ}$; GDL surface contact angle, $140^{\circ}$; side pipe diameter, $50 \mu \mathrm{m}$ ).

at the low water flow rate. This periodic flow feature is not present when the water flow rate is large. In contrast, a continuous conducting pathway for the liquid water flow is formed along the GC corner as shown in Fig. 10f. So, we conclude that the periodic feature of the film flow at the GC corners cannot be captured with a high water flow rate. However, both Fig. 10 and Fig. 11 show that the gas drag force is quite important for the film flow, as it enhances the liquid water removal. So, when formulating governing equations for water flow in the GC, the gas drag force has to be taken into account besides the capillary forces.

Since higher gas flow rate corresponds to larger gas drag force for the same film flow condition, practically, we can increase the gas flow rate to enhance the film removal along the GC corners. The effect of the gas flow rate on the film flow along the GC corner is present in Fig. 12 in terms of the water saturation in the whole GC as a function of time. Three gas flow rates are used with Re numbers of 150, 200, and 250. Before the liquid water flows out of the GC, we have the same water saturation for the three cases due to the same water flow

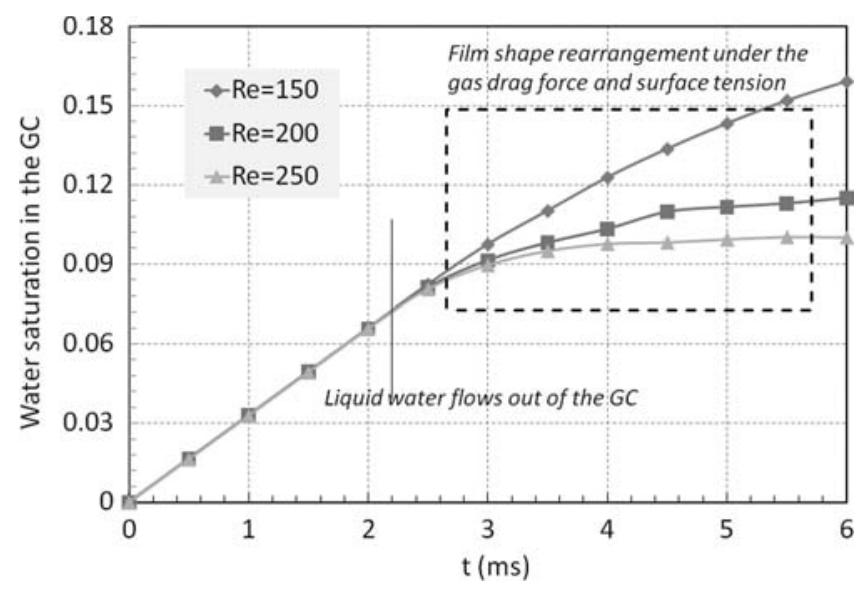

Figure 12. Effect of the Gas flow rate on the film flow at the corner of the GC (water saturation in the GC vs. flow time; case 8-10). 


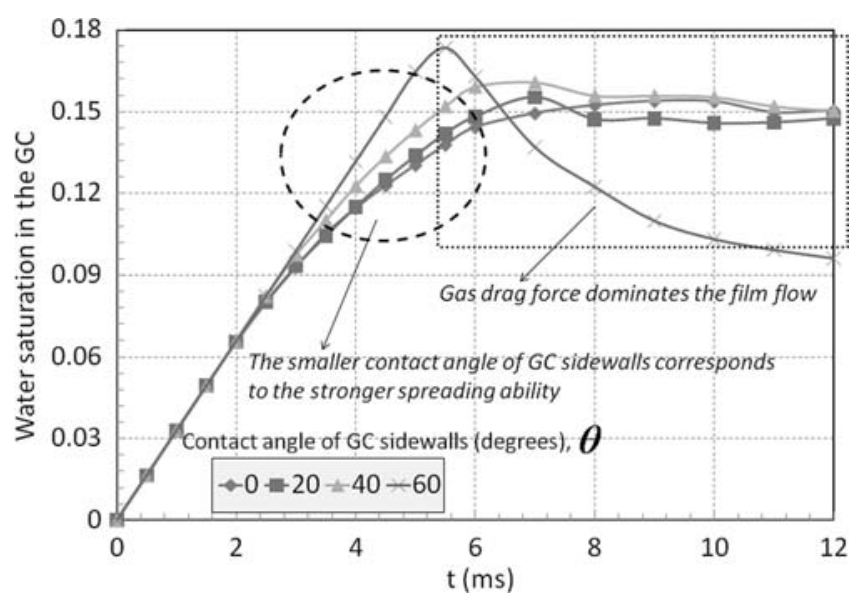

Figure 13. Effect of the sidewall contact angle on the film flow along the corner of the GC (water saturation in the GC vs. flow time; case 8 and case 11-13).

rate from the side pipe. After the breakthrough of the liquid water at the GC outlet, there is a distinct difference between the three cases; the saturation increase is slower for higher Re number. This is because the film shape is rearranged differently under the gas drag and surface tension forces for each case. One should note that the flow conditions are still unsteady even at $\mathrm{t}=6 \mathrm{~ms}$. For the lower two curves $(\mathrm{Re}$ $=200,250$ ), the flow conditions can be assumed to be very close to the steady state according to the water saturation tendency. It is obvious that increasing the gas flow rate can mitigate the film flooding at the GC corners. However, it needs to keep in mind that higher gas flow rate gives rise to higher pressure loss along the GC, which means a lower cell efficiency.

A similar study is performed for determining the effect of sidewall contact angle on the water flooding in the GC. Results are shown in Fig. 13 in terms of change of water saturation in the whole GC with time for four different sidewall contact angles, namely, 0, 20, 40, and 60 degrees. For the contact angle of 60 degrees, the water saturation in the $\mathrm{GC}$ reaches its peak value of 0.17 at about $\mathrm{t}=5.5 \mathrm{~ms}$. After that, it starts to go down quickly, the curve slope becomes smaller and smaller. This whole process indicates that the gas drag force suppresses the increase of the film thickness, and tries to balance the viscous resistance from the sidewall. At the end of the simulation, the water saturation in the GC approaches a certain value. From the region enclosed by a dashed circle in Fig. 13, we can see that the water saturation in the GC is significantly affected by the sidewall contact angle. The water saturation in the GC decreases with the decrease of the sidewall contact angle. This is attributed to the fact that the GC sidewall with a smaller contact angle can spread out the liquid water faster. So, for the GC sidewall with the contact angle of zero, it can spread most liquid water out of the GC, leading to the smallest water saturation. The flow region enclosed by a dashed rectangle denotes the regime that the gas drag force dominates the film flow; the film shapes are also rearranged during this period (Fig. 10g). At the same time, the capillary force starts to resist the water film flow along the GC corner. The contact angle of 60 degrees corresponds to a small capillary force; as a result, the water saturation in the GC reduces considerably with time as shown in Fig. 13. In contrast, the water saturation for the other three contact angles does not change much with time. We also find that the water saturation evolution for the contact angle of 60 degrees is quite different from the others, which may be explained by the fact that the film flow is very unstable when the contact angle of the sidewall is larger than 45 degrees in the rectangular corner of the GC (Concus and Finn condition ${ }^{29}$ ). In PEFC applications, normally the water removal ability is evaluated over a large time scale (i.e. several hours). Based on this point, we conclude that a sidewall with less hydrophilicity is advantageous to reducing the film flooding along the GC corners

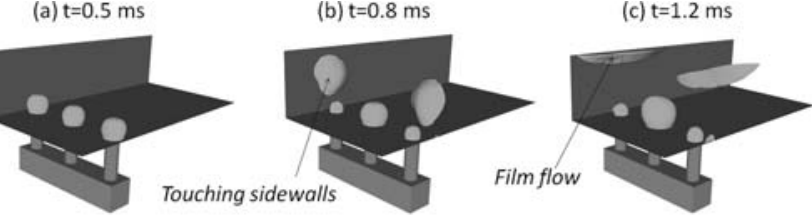

(d) $\mathrm{t}=1.8 \mathrm{~ms}$ (e) $\mathrm{t}=2.5 \mathrm{~ms}$

(f) $\mathrm{t}=3.5 \mathrm{~ms}$
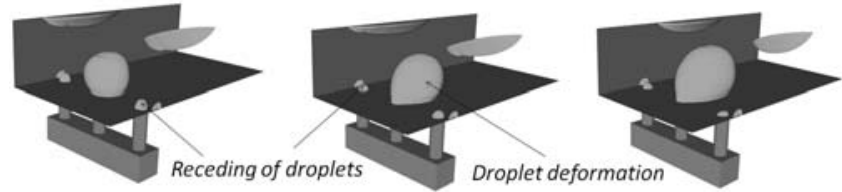

(g) $\mathrm{t}=4.0 \mathrm{~ms}$

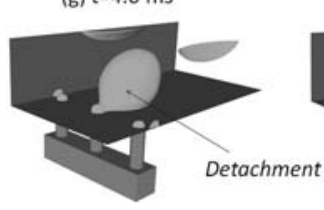

(h) $\mathrm{t}=4.5 \mathrm{~ms}$

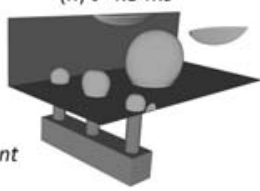

(i) $\mathrm{t}=5.0 \mathrm{~ms}$

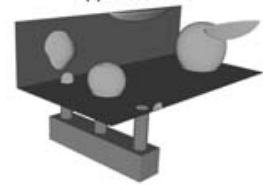

Figure 14. Water dynamics in the GC, filling by three pipes with the same diameter (case 15: gas Re number, 150; water flow rate, $2.0 \times 10^{-6} \mathrm{~kg} \mathrm{~s}^{-1}$; sidewall contact angle, $40^{\circ}$; GDL surface contact angle, $140^{\circ}$; side pipes diameter, $50 \mu \mathrm{m}$; middle pipe diameter, $50 \mu \mathrm{m}$ ).

(Fig. 13). However, we must note that a sidewall with less hydrophilicity is incapable of removing the water clogging (Fig. 3e) immediately maybe occurred in the GCs of an operating PEFC. This would result in the non-uniform distributions of the reactants in the cell, ${ }^{30}$ and reduce the cell performance. So, in practice, a proper contact angle of the GC sidewalls should be selected to balance both requirements, namely, increasing the film removal ability (less hydrophilicity) and removing the water clogging fast (higher hydrophilicity).

Competition between film and droplet flows. - Under the water flooding situation of the cathode side of a PEFC, several conducting pathways for the liquid water transport are formed in the fibrous GDL. It has been observed experimentally that small droplets preferentially emerge from certain pores on the GDL surface. Depending on the locations of such pores, we could have both film and droplet flows. On the other hand, these conducting pathways in the GDL are not isolated, but maybe connected by several water reservoirs inside the GDL and CL. So, it is obvious that these conducting pathways would compete to feed the liquid water into the GC. As stated above, we simplify the conducting pathways by three cylindrical pipes, which are connected to the same water reservoir within the CL (Fig. 2a).

Fig. 14 shows the liquid water dynamics in the GC. The liquid water is delivered by three cylindrical pipes with the same diameter of $50 \mu \mathrm{m}$. At $\mathrm{t}=0.5 \mathrm{~ms}$, three droplets with the same size form on the hydrophobic GDL surface. After a while, when two side droplets are big enough to touch the sidewalls, the water spreads out quickly and forms the film flow along the corners as shown in Fig. 14b and $14 \mathrm{c}$. Due to the small water flow rate from the side pipes, we observe the discontinuities of the water flow at the corners, which is already discussed in the section on film dynamics. From Fig. 14c to Fig. 14f, we observe that the middle droplet becomes bigger and bigger, while two side droplets recede back into the pipes. This receding phenomenon implies that more liquid water is filled into the GC through the middle pipe, since three pipes are connected together with the fixed water flow rate from the CL. In Fig. 14b, after wicking into the film at the corners, the two side droplets become much smaller than the middle one. As a result, the bigger middle droplet has much smaller capillary pressure over the interface. If we assume the same gas pressure around three droplets and uniform liquid water pressure inside each droplet, we can conclude that the liquid water pressure inside the middle droplet is much smaller than those in two side droplets. So, the driving force for the water flow through the 

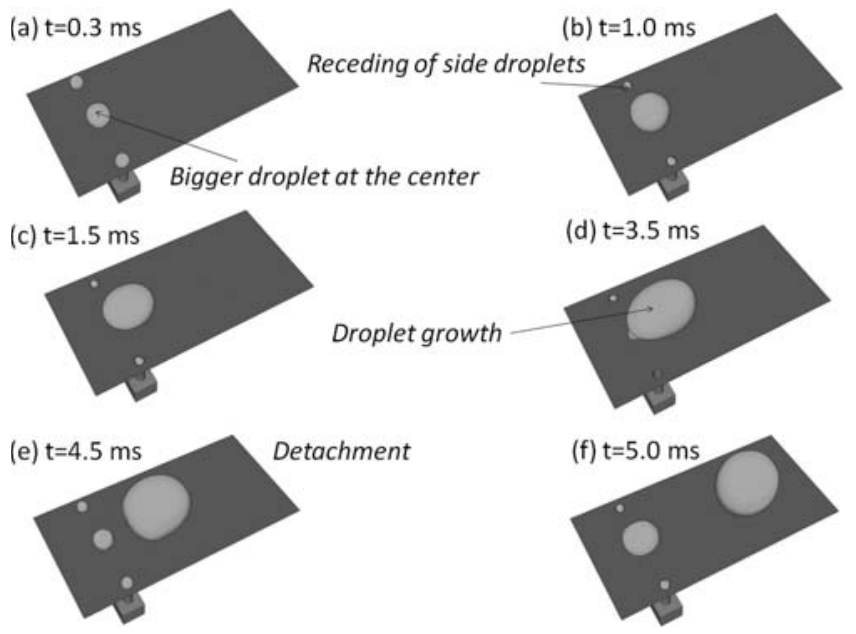

Figure 15. Water dynamics in the GC, filling by three pipes with different diameters (case 16: gas Re number, 150; water flow rate, $2.0 \times 10^{-6} \mathrm{~kg} \mathrm{~s}^{-1}$; sidewall contact angle, $40^{\circ}$; GDL surface contact angle, $140^{\circ}$; side pipes diameter, $40 \mu \mathrm{m}$; middle pipe diameter, $50 \mu \mathrm{m}$ ).

middle pipe is biggest, and this can explain the aforementioned receding phenomenon. At $\mathrm{t}=4.0 \mathrm{~ms}$ (Fig. 14g), the middle droplet reaches its critical size, and is detached by the gas drag force. From $\mathrm{t}=4.5 \mathrm{~ms}$ (Fig. 14h), a second water flow cycle starts as shown in Fig. 14i. So, for this kind of conducting pathway settings, we could have the droplet detachment, side droplets receding, and film filling at the corners cyclically. To some extent, this numerical study resembles the processes observed in some experiments. ${ }^{19,20}$

In the GDL, the sizes of the conducting pathways are randomly formed maybe due to the random structure of the GDL. In what follows, we investigate the effect of the sizes of the conducting pathways on the competing water filling into the GC. In Fig. 15, we reduce the diameter of the side pipes to $40 \mu \mathrm{m}$, while the middle pipe has an unchanged diameter of $50 \mu \mathrm{m}$. At the beginning, a relatively bigger middle droplet is observed as expected due to the smaller flow resistance (Fig. 15a). With the increase of the middle droplet, the driving force for the water flow through the middle pipe is bigger and bigger, compared to that through the side pipes. So, the two side droplets recede back into the pipes quickly, whereas the middle droplet grows up with time. At about $\mathrm{t}=4.5 \mathrm{~ms}$, the middle droplet is detached by the gas flow, and later the second cycle starts. For this case, the film flow along the corners does not occur because of the higher flow resistance for the two side pipes. Only periodic droplet detachment is found. In Fig. 16, we change the middle pipe diameter to $40 \mu \mathrm{m}$, and the diameter of the side pipe is set to $50 \mu \mathrm{m}$. Then, a totally different water dynamics in the GC is observed. In contrast to Fig. 15a, two bigger droplets form nearby the sidewalls due to the smaller water flow resistance as shown in Fig. 16a. After touching the hydrophilic sidewalls, the surface tension force assists in the water flow from the side pipes, while the middle droplet recedes back into the pipe immediately. It is interesting to find that the isolated film flow at the left corner is formed, while a conducting pathway for the water transport along the right corner is established. This may be caused by the highly unstable flow condition in the GC. It must be noted that in real water flooding of PEFC applications, we never obtain continuous conducting pathways for the liquid water transport along the GC corners, since the water flow rate is extremely low. This is also explained in the section on film dynamics. After a conducting pathway is formed as in Fig. 16c, the liquid water flow through the remaining two pipes (middle and left ones) is totally suppressed, because the surface tension effect on the film flow at the right corner is much larger, resulting in a quite big driving force for the water flow through the right pipe. In addition, we obtain asymmetrical water dynamics in the GC as shown in Fig. 16, even though the GC with symmetrical structure is used. We attribute

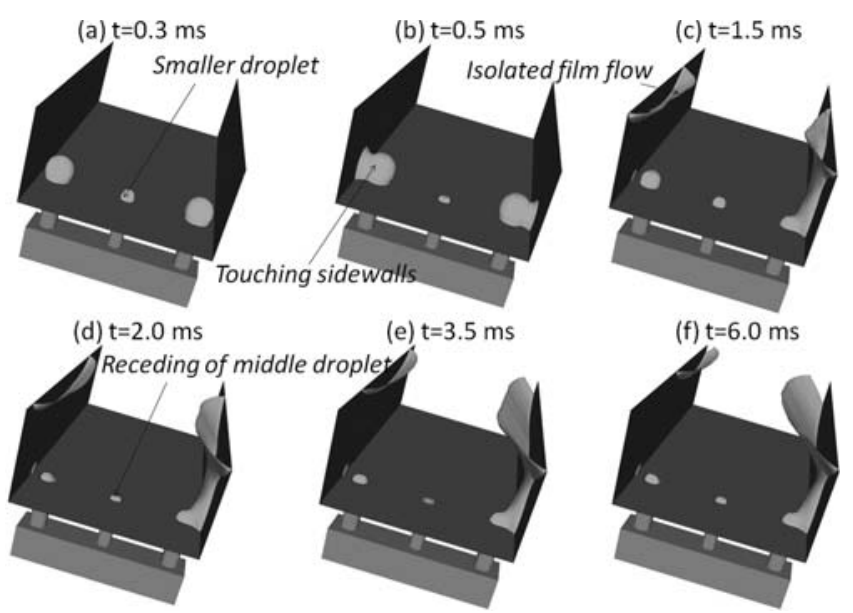

Figure 16. Water dynamics in the GC, filling by three pipes with different diameters (case 17: gas Re number, 150; water flow rate, $2.0 \times 10^{-6} \mathrm{~kg} \mathrm{~s}^{-1}$; sidewall contact angle, $40^{\circ}$; GDL surface contact angle, $140^{\circ}$; side pipes diameter, $50 \mu \mathrm{m}$; middle pipe diameter, $40 \mu \mathrm{m}$ ).

it to the physical phenomenon of liquid water dynamics in the GC, due to its transient and unstable flow features. However, as demonstrated in Fig. 11, due to the small water flow rate in an operating PEFC, we cannot have continuous conducting pathways for the liquid water transport along the GC corners. Instead, periodic film flow (or slugs) at both corners can be expected, meanwhile droplet dynamics is always suppressed in this case.

\section{Conclusions}

In this work, based on a novel geometrical setup, a series of direct simulations (VOF) are conducted to explore in detail the liquid water dynamics in a GC, including the droplet dynamics, film dynamics, and the competition between the film and droplet flows. The main findings and conclusions are summarized as follows:

(1) With the increase of the droplet size in the GC, the three-phase contact line extends along both the gas flow and transverse directions to resist the increased gas drag force. For the same flow conditions of a droplet, before its detachment, the contact line deformation increases with increasing the gas flow rate, and decreases as reducing the hydrophobicity of the GDL surface. A slightly hydrophobic GDL surface could lead to an early droplet detachment. However, it results in large water coverage over the GDL surface, which would hinder reactants diffusion into the reactive sites. We also find that too highly hydrophobic GDL surface is unnecessary for the droplet removal in the GC.

(2) As filling the liquid water into the GC through the side conducting pathway (nearby one hydrophilic GC sidewall), the film flow forms along the GC corner with the help of the surface tension force. At the beginning, the surface tension dominates the film flow, so, the liquid water spreads over the corner quickly. The film thickness distribution is from the thick to the thin, from the inlet position to the GC outlet. With more and more liquid water accumulating in the $\mathrm{GC}$, the gas drag force starts to play an importance role. Finally, we get an opposite film thickness distribution, which is from the thin to the thick along the GC corner. Increasing the gas flow rate can mitigate the film flooding at the GC corner. However, it generates high pressure loss along the GC. Practically, a proper contact angle of the GC sidewalls should be selected to balance two requirements, namely, increasing the film removal ability (less hydrophilicity) and removing the water clogging fast (higher hydrophilicity).

(3) The sizes of conducting pathways formed in the GDL have a big impact on the water dynamics in the GC. For different 
combinations of pipe diameters, we could have the droplet detachment, droplet receding, and isolated film flow at the corner cyclically, or only have the film flow at the corners and the droplet flow at the center. The surface tension forces from the hydrophilic sidewalls and the interface between the droplet and gas both play important roles in the competing water flow from the conducting pathways into the GC. These competition mechanisms could give us the possibility to regulate liquid water flow into GCs, and contribute to the water management in GCs.

\section{References}

1. M. M. Mench, Fuel Cell Engines, John Wiley \& Sons, 2008.

2. G. Hoogers, Fuel Cell Technology Handbook, CRC Press, 2003.

3. C. Y. Wang, Chem. Rev., 104, 4727 (2004).

4. H. Li, Y. H. Tang, Z. W. Wang, et al., J. Power Sources, 178, 103 (2008)

5. X. G. Yang, F. Y. Zhang, A. L. Lubawy, and C. Y. Wang, Electrochem. Solid-State Lett., 7, A408 (2004).

6. E. C. Kumbur, K. V. Sharp, and M. M. Mench, J. Power Sources, 161, 333 (2006)

7. A. Bazylak, Int. J. Hydrogen Energy, 34, 3845 (2009).

8. R. Mukundan and R. L. Borup, Fuel Cells, 9, 499 (2009).

9. S. H. Ge and C. Y. Wang, J. Electrochem. Soc., 154, B998 (2007).

10. F. Y. Zhang, X. G. Yang, and C. Y. Wang, J. Electrochem. Soc., 153, A225 (2006).

11. I. S. Hussaini and C. Y. Wang, J. Power Sources, 187, 444 (2009).
12. A. Theodorakakos, T. Ous, M. Gavaises, J. M. Nouri, N. Nikolopoulos, and H. Yanagihara, J. Colloid Interface Sci., 300, 673 (2006).

13. Y. H. Cai, J. Hu, H. P. Ma, B. L. Yi, and H. M. Zhang, J. Power Sources, 161, 843 (2006).

14. D. Rensink, J. Roth, and S. Fell, Liquid water transport and distribution in fibrous porous media and gas channels, Proc. 6th Int. ASME Conf. On Nanochannels, Microchannels and Minichannels (2008), ICNMM2008-62087.

15. X. Zhu, P. C. Sui, and N. Djilali, J. Power Sources, 181, 101 (2008).

16. L. Hao and P. Cheng, J. Power Sources, 190, 435 (2009).

17. J. Chio and G. H. Son, J. Mech. Sci. Technol., 22, 2590 (2008).

18. K. S. Chen, M. A. Hickner, and D. R. Noble, Int. J. Energy Res., 29, 1113 (2005).

19. A. Bazylak, D. Sinton, and N. Djilali, J. Power Sources, 176, 240 (2008).

20. C. Hartnig, I. Manke, R. Kuhn, S. Kleinau, J. Goebbels, and J. Banhart, J. Power Sources, 188, 468 (2009).

21. J. H. Nam and M. Kaviany, Int. J. Heat Mass Transf., 46, 4595 (2003).

22. S. Lister, D. Sinton, and N. Djilali, J. Power Sources, 154, 95 (2006).

23. O. Ubbink and R. I. Issa, J. Comput. Phys., 153, 26 (1999).

24. C. W. Hirt and B. D. Nichols, J. Comput. Phys., 39, 201 (1981).

25. K. Jiao, B. Zhou, and P. Quan, J. Power Sources, 154, 124 (2006).

26. S. Whitaker, The method of volume averaging, Kluwer Academic Publishers, 1999

27. J. U. Brackbill, D. B. Kothe, and C. Zemach, J. Comput. Phys., 100, 335 (1992)

28. FLUNET6.3, User's Guide, Fluent Inc., 2006.

29. P. K. Sinha and C. Y. Wang, Electrochim. Acta, 52, 7936 (2007)

30. S. Basu, J. Li, and C. Y. Wang, J. Power Sources, 187, 431 (2009).

31. L. Hao and P. Cheng, J. Power Sources, 195, 3870 (2010).

32. P. P. Mukherjee, C. Y. Wang, and Q. Kang, Electrochim. Acta, 54, 6861 (2009). 